\title{
Obesity and chronic inflammation crosslinking
}

\author{
DOROTA ARTEMNIAK-WOJTOWICZ, ANNA M. KUCHARSKA, BEATA PYRŻAK \\ Department of Paediatrics and Endocrinology, University Clinical Center, Children's Hospital in Warsaw, Poland \\ Department of Paediatrics and Endocrinology, Medical University of Warsaw, Poland
}

\begin{abstract}
The adipose tissue has been recognized as an active organ involved in numerous metabolic, hormonal and immunological processes. Obesity and associated chronic inflammation leads to many metabolic and autoimmune disorders. The number of cells, their phenotype and distribution in adipose tissue depends on the degree of obesity. Polarization of macrophages towards M1, neutrophils influx to adipose tissue, activation of Th1 and Th17 cells and increased level of proinflammatory cytokines are characteristic for obesity-induced inflammation. Several mechanisms, such as adipocytes' hypoxia, oxidative stress, endoplasmic reticulum stress, impairment of PPAR receptors, inflammasomes' activation and activation of TLR are involved into development of chronic obesity-induced inflammation. A better understanding of this processes can provide new treatments for obesity and related disorders.
\end{abstract}

Key words: obesity, inflammation, children.

(Centr Eur J Immunol 2020; 45 (4): 461-468)

\section{Introduction}

Obesity and its complications an increasing problem in developed countries. In 2011, about 500 million people worldwide suffered from obesity (body mass index (BMI) $>30 \mathrm{~kg} / \mathrm{m}^{2}$ ), and this figure is estimated to increase by at least double by 2030. Basing on many studies the adipose tissue (AT) has been recognized as an active organ involved in numerous metabolic, hormonal and immunological processes, affecting other organs and systems playing an important role in whole-body homeostasis. There are several mechanisms proposed to explain how the excessive

Table 1. Typical changes of lymphatic cell's profile in VAT in obese individuals basing on animal models and human subjects studies

\begin{tabular}{lcc}
\hline Type cells & $\begin{array}{c}\text { Alterations of immune cells } \\
\text { contribution in obese in } \\
\text { comparison to lean individuals }\end{array}$ & References \\
\hline Macrophages M1 & $\uparrow$ & {$[4]$} \\
\hline Macrophages M2 & $\downarrow$ & {$[4]$} \\
\hline Neutrophils & $\uparrow$ & {$[12]$} \\
\hline Eosinophils & $\downarrow$ & {$[16]$} \\
\hline Natural killer & $\uparrow$ & {$[19]$} \\
\hline Th1 cells & $\uparrow$ & {$[2]$} \\
\hline Th17 cells & $\uparrow$ & {$[21]$} \\
\hline Th2 cells & $\downarrow$ & {$[2]$} \\
\hline Treg cells & $\downarrow$ & {$[23]$} \\
\hline B cells & $\uparrow$ & {$[26]$} \\
\hline ILCs2 & $\downarrow$ & {$[28]$} \\
\hline$\uparrow$ increased, $\downarrow$ decreased &
\end{tabular}

AT can cause metabolic disorders. One of the most promising concepts includes the development of local and systemic inflammation characterized by increased infiltration of AT by immune cells and increased production of circulating proinflammatory factors. This process is chronic and has much smaller amplitude than inflammation caused by infection, damage or cancer. Despite lower intensity a sterile, obesity-induced inflammation has a profound effect on metabolic pathways [1]. Long-term, low-grade inflammation may prone fatty liver disease, cardiovascular disease, type 2 diabetes, asthma, neurodegeneration and some types of cancer [2]. The involvement of inflammation in the development of insulin resistance (IR) is also well documented, as well as predisposition to autoimmune disorders, such as psoriasis and inflammatory bowel disease [3].

\section{Adipose tissue as the milieu of immune cells}

Secretory activity of AT varies and is determined by its cellular composition. The number of cell types, their phenotype and distribution in AT depends on the degree of obesity (Table 1). Increasing obesity leads to fat remodelling and changes in the AT microenvironment.

\section{AT immune cells in obesity}

\section{Macrophages}

AT macrophages (ATMs) represent the largest subpopulation of AT immune cells, encompassing $4 \%$ of normal visceral fat with an increase to $12 \%$ in excessed adiposity [4]. In rodent models [5] as well as in humans [4] obesi-

Correspondence: Assoc. Prof. Anna Małgorzata Kucharska, Department of Paediatrics and Endocrinology, University Clinical Center, Children's Hospital in Warsaw, e-mail: ankucharska@wum.edu.pl

Submitted: 21.08.2020; Accepted: 26.10.2020 
ty is associated with increased ATMs which are the main source of cytokines. ATMs infiltration correlate positively with stromal-vascular expression of proinflammatory factors, including tumor necrosis factor alpha (TNF- $\alpha$ ), inducible nitric oxidase synthase (iNOS), and inhibitors of $\kappa \mathrm{B}$ kinase $\beta$ (IKKB) [6]. Macrophages infiltrating AT in obese individuals are mostly of outside origin, from systemic circulation. The mechanism of their recruitment is only partially elucidated. Adipocyte hypertrophy and necrosis, tissue hypoxia, metabolic endotoxemia and endoplasmic reticulum (ER) stress are suggested as initiators of macrophage infiltration. The crucial role of attracting circulating monocytes into AT is dependent on complex network of chemotactic cytokines (chemokines) and adipokines secreted from the AT and their corresponding receptors on the attracted immune cells. Beside the number of ATMs also their function and tissue localization are different in obesity. Macrophages are divided into two subpopulation types: a classically activated M1 type and the alternatively activated M2 type. M2 occur in lean's subjects adipose tissue, while M1-type predominate in obese individuals. Polarization between macrophage types is observed in inflammatory processes and also in some type of cancer [7]. Activated M1 macrophages are characterised by increased production of proinflammatory cytokines: interleukin (IL)-6, TNF- $\alpha$, IL-12, IL-23, and reduced synthesis of anti-inflammatory IL-10 [8]. Activation of M1 macrophages occurs through cytokines produced by type 1 helper lymphocytes (Th1), while type 2 helper cells (Th2) stimulate M2. M2 macrophages are involved in the repair of damaged tissues and also prevent the development of inflammation by producing in large quantities IL-10, with a simultaneous decrease in IL-12 and IL-23 synthesis [9]. It has been initially suggested that there is an M2-to-M1 phenotypic switch in AT in obesity [6]. In vivo, there are probably no such clear-cut M1-versus-M2 states, but rather a dynamic continuum of the M1-M2 spectrum depending on the local cytokine microenvironment, and most macrophages are somewhere between an M1 and M2 phenotype. In $\mathrm{M} 2$ are transcribed genes of proteins such as arginase1, macrophage mannose receptor 1 and IL-1 receptor antagonist, which are regulated by receptors activated by peroxisome proliferator-activated receptor (PPAR). Thus, PPAR regulates the polarization of macrophages towards M2 and supresses the expression of proinflammatory cytokine genes [10], while interferon gamma (IFN- $\gamma)$ is a potent inducer of M1 polarization [11].

\section{Neutrophils}

Neutrophils are involved in innate immunity and are regarded as the first immune cells responding at sites of inflammation. They are first immune cell infiltrating visceral adipose tissue (VAT) in obesity, and initiating AT inflammation. Neutrophils in VAT are thought to be primarily of peripheral origin not from fat-resident cells. A possible trigger factor of the neutrophils' activation could be the increased plasma concentration of leptin and TNF- $\alpha$ observed in morbid obesity. Leptin can activate neutrophils via indirect induction of TNF- $\alpha$ secretion by monocytes. A high fat diet (HFD) causes changes in AT composition. In mice after 3 days of HFD, an increased proportion of neutrophils was seen in VAT, and they maintained up to 12 weeks in comparison to normal diet-fed mice [12]. Neutrophils were also found in the AT of lean mice, although they represent only a small fraction $(<1 \%)$ of all AT immune cells [13]. Neutrophil to lymphocyte ratio (NLR) seems to be costeffective marker for the detection of subclinical inflammation, which correlates with C-reactive protein (CRP) levels [14], nevertheless not all authors confirmed a correlation between NLR and obesity in children [15].

\section{Eosinophils}

Eosinophils produce a vast array of Th- 2 cytokines like transforming growth factor beta (TGF- $\beta$ ), IL-13, IL-10 and IL-4, which participate in anti-inflammatory immune responses, macrophage polarization towards M2 and differentiation of Th2 cells [16]. In mice models, adiposity decreased AT eosinophil numbers leading to reduced insulin sensitivity, while the increase in eosinophil numbers due to helminth infection or the overexpression of IL-5 improved obesity-induced IR [17].

\section{Natural killer (NK)}

NK are the next kind of immune cells present in AT. In the response to HFD in mice the number of NKT cells strongly increases [18]. NK cells have been shown to drive M1 polarization through the production of IFN- $\gamma$ [19]. In turn, invariant-chain natural killer (iNKT) cells sustain M2 ATMs in VAT. They are present in relatively high amount in AT in lean subjects [20]. Lack of iNKT cells in mice (as a result of genetic deficiency for J $\alpha 18$ or CD1d deficiency) leads to reduction of IL-4 and IL-13 in AT and increase pro-inflammatory ATMs.

\section{Adaptive immune cells in obesity}

\section{T cells}

Numerous studies have investigated the role of T-cells in the obesity-induced inflammation. The CD4+T cells could be subdivided into four main groups: proinflammatory Th1 subset producing INF- $\gamma$, and Th17 subset synthesizing IL-2, IL-17, IL-22; anti-inflammatory Th2 subset releasing IL-13, IL-4, IL-5 and T-regulatory cells (Treg) producing IL-10, TGF- $\beta$. In mice model Th1 and Th 2 cells in leans, are present in nearly equal amounts, and over half of the CD4+T cells are Treg cells [21]. In obesity AT composition changes, number of proinflammatory cells increases and the ratio of Th1 to Th2 increases significantly [2]. Increased number of Th1 cells secreting large amount 
of INF-r, promote M1 polarization of ATMs and exert AT inflammation and IR. In the obesity T cells infiltrate AT early, even before a major inflow of macrophages. Th17 lymphocytes and their IL-17 are found in AT, but at much lower level than Th1 cells. Their level increases with development of obesity and the increasing IL-17 production is reported as associated with glucose intolerance in type 2 diabetes [22]. In concert with this finding are the observation in HFD-fed mice with IL-17 deficiency, in which the weight gain did not impair glucose tolerance and insulin sensitivity, suggesting the pathogenic role of IL-17 in glucose homeostasis [23]. In contrast, Treg cells prevent the development of chronic inflammation in VAT. Amount of Tregs is high in lean AT, and low in obese AT. Obesity is linked with a reduction of Treg cells in abdominal AT, but not subcutaneous tissue [2, 21, 24, 25]. Depletion of Treg in wild-type lean mice increases levels of insulin and proinflammatory cytokines both in the circulation and in the AT [21]. Treg cells are known to supress proinflammatory responses and promote M2 polarization of ATMs by producing of IL-4, IL-10 and IL-13 [26]. It was found, that CD8+cells increase a three- to fourfold in AT in obese mice in comparison to lean mice [2]. Increased number of CD8+ $\mathrm{T}$ cells promote macrophage infiltration into AT after eight to ten weeks of HFD. CD8+T cells also promote monocyte differentiation into M1 macrophages [25]. It was shown, that mice deficient for $\mathrm{CD} 8+\mathrm{T}$ cells have fewer fat macrophages with reduced numbers of proinflammatory cytokines such as TNF- $\alpha$ and IL-6 [25]. In contrast, the adoptive transfer of CD8+T cells into obese mice leads to increased infiltration of M1 macrophages and worsens glucose tolerance and insulin sensitivity [25].

\section{B cells}

B cells are principal in humoral immunity. Recent investigations also showed that the number of $B$ cells increases in AT in the course of obesity, which is associated with elevated concentrations of immunoglobin G2c (IgG2c) both in circulation and AT [27]. In mice upon four weeks of HFD, proinflammatory B cells infiltrate AT before $\mathrm{T}$ cells, and through the production of pathogenic $\mathrm{IgG}$ antibodies modulate function of T cells and promote M1 polarization of ATMs. Moreover B cell depletion improve insulin sensitivity and IgG2c antibodies are elevated in obese mice [27]. The IgG2c transfer into lean animals is resulting in development of AT inflammation and IR. Human B cells are also reported to promote proinflammatory cytokine production by Th17 cells during adiposity [27].

\section{Innate lymphoid cells type 2 (ILCs2)}

ILCs2 play a role in lymphoid tissue development, and in the response to helminths and allergy [28]. Recently it was discovered, that they are able to is produce IL-5 and IL-13, and prone eosinophil and M2 macrophage accumulation in VAT [28]. Decline numbers of eosinophils and
M2 macrophages in AT in obese humans and mice are accompanied with reduced number of ILC2s [29].

\section{Cytokines milieu in obesity}

In the context of AT inflammation, we distinguish adipokines with pro- and anti-inflammatory effects. The best known proinflammatory adipokines are: TNF- $\alpha$, IL-6, leptin, visfatin, resistin, angiotensin II, and plasminogen activator inhibitor 1; they are released mainly by obese AT. In contrast, AT from lean subjects mostly secrets anti-inflammatory cytokines such as TGF- $\beta$, adiponectin, IL-10, IL-4, IL-13, IL-1 receptor antagonist (IL-1Ra), and adipolin. In this review, we will discuss the best known cytokines that play a role in the development of obesity-induced inflammation.

\section{Tumor necrosis factor-alpha (TNF- $\alpha$ )}

TNF- $\alpha$ is primarily secreted by macrophages, and in smaller amount by fibroblasts, neutrophils, lymphocytes, as well as adipocytes. By activating mitogen-activated protein kinases (MAPK) and the nuclear factor kappa B $(\mathrm{NF} \kappa \mathrm{B})$ signalling pathways, TNF- $\alpha$ affects the release of other proinflammatory cytokines such as IL-6 and IL-1 $\beta$. Furthermore, TNF- $\alpha$ downregulates the mRNA level of adiponectin. Expression of TNF- $\alpha$ and one of its receptors has been shown to increase in plasma and AT of obese individuals, and correlates positively with increased BMI [30]. It was documented that adipose tissue in obese mice secretes TNF- $\alpha$, and adipocyte-derived TNF- $\alpha$ directly influences obesity-induced IR [31]. This was the first functional link between obesity and inflammation and over the years it has evolved into the concept of "metabolic inflammation". Of importance, there are evidences that circulating level of TNF- $\alpha$ reduces with weight loss [32].

\section{Interleukin-6 (IL-6)}

IL-6 is secreted by AT, skeletal muscle, and the liver. About one-third of total circulating IL-6 in healthy individuals derives from adipocytes [33]. IL-6 expression increases in AT and plasma of obese subjects, and correlates positively with BMI, waist circumference, and free fatty acid levels [34]. IL-6 strongly stimulates hepatocytes to produce and secrete C-reactive protein (CRP), and promotes the development of inflammation. Study in adolescents showed that the IL-6 increased with increased BMI independently on sex [35]. Furthermore, the proinflammatory effect of IL-6 is augmented by the simultaneous suppression of adiponectin in human adipocytes [34].

\section{C-reactive protein (CRP)}

CRP is an acute-phase protein and the best known sensitive marker for systemic inflammation. The highest value in obesity has high-sensitivity C-reactive protein (hs-CRP). An increased level of CRP is widely observed in obese hu- 
mans. In study in overweight children, the CRP concentration was significantly elevated, compared to normal weight children [36] and correlates positively with body weight and BMI [37]. CRP is synthesized and secreted primarily in human hepatocytes, and its synthesis is regulated mainly by IL- 6 and IL-1. The release of IL- 6 from adipose tissue may induce elevated CRP concentrations with increase of body fat. Raised hs-CRP levels suggest a chronic inflammatory process, and can be used as predictor of metabolic and cardiovascular disorders [38].

\section{Leptin}

Leptin is mainly secreted by adipocytes, and its circulating levels are proportional to fat cell mass. Leptin is essential to control appetite and regulate energy homeostasis, and several neuroendocrine axes by specific leptin receptors in the hypothalamus and other organ [39]. Leptin also plays an important role in controlling immunity and development of AT inflammation by recruiting neutrophils and activation of NK cells and macrophages [40]. This effect is related to stimulating proliferative responses of T-cell and polarizing naive $\mathrm{CD} 4^{+} \mathrm{T}$-cell proliferation toward the Th1, resulting in a marked increase in Th1-type cytokine production. It also induces the expression of proinflammatory cytokines by macrophages and monocytes, and acts directly on hepatocytes to promote CRP expression. Leptin activates also human B lymphocytes to secrete TNF- $\alpha$, IL-6, and IL-10 through the JAK2, STAT3, p38MAPK, and ERK signalling pathways, and sustains Th17 pro-inflammatory function [26].

\section{Resistin}

Resistin in humans is primarily secreted by monocytes and macrophages with some contribution from adipocytes. The name of this cytokine is the result of its ability to resist the action of insulin. Circulating resistin levels are increased in obese humans, which is probably associated with increased infiltration of macrophages in the AT [41]. Resistin by binding toll-like receptor 4 (TLR4) on human leukocytes stimulates the secretion of several pro-inflammatory cytokines, like IL-12, IL-6, IL-1 $\beta$, and in turn, these molecules further enhance resistin expression. Resistin can induce a pro-inflammatory response from adipocytes (TNF- $\alpha$, IL-6, MCP-1) [42].

\section{Angiopoietin like protein 2 (ANGPTL2)}

ANGPTL2 is secreted by adipocytes, and its' plasma concentration correlates positively with obesity, IR markers and CRP level in humans [43]. ANGPTL2 deficiency has been shown to reduce inflammation and decrease secretion of proinflammatory cytokines in diet-induced obese mice [43]. Additionally, ANGPTL2 through the activation of integrin signalling, stimulates inflammatory responses by monocytes, macrophages and endothelial cells [43].

\section{Anti-inflammatory adipokines}

\section{Adiponectin}

Adiponectin, one the most intensively investigated anti-inflammatory adipokine, is secreted predominantly by adipocytes. Rodent gain- and loss-of-weight models show that adiponectin level is inversely correlated with visceral fat accumulation, and the degree of AT inflammation [44]. Its levels in the plasma and AT are decreased in obese subjects compared with lean ones [44]. Adiponectin is expressed at the highest levels by the functional adipocytes in lean organisms. Its expression is downregulated in the dysfunctional adipocytes characteristic for obesity. Adiponectin secretion in adipocytes is stimulated by the activation of PPARr [45], whereas its production is inhibited by pro-inflammatory factors, like TNF- $\alpha$, IL- 6 , and also by hypoxia and oxidative stress [46]. Adiponectin supresses TNF- $\alpha$ production in obese mice, and adiponectin-deficient mice develop insulin resistance with high levels of TNF- $\alpha$ in AT [47]. Plasma adiponectin levels are negatively correlated with CRP levels in obese and diabetic patients, and its low levels are associated with higher CRP levels in non-diabetic or healthy subjects [45]. Adiponectin also inhibits Toll-like receptor-mediated NF- $\kappa \mathrm{B}$ activation in mouse macrophages [48] and stimulates the production of the anti-inflammatory cytokine IL-10 by human macrophages. Study of macrophages and AT stromal vascular fraction cells of adiponectin-deficient mice, show increased expression of pro-inflammatory M1 and decreased expression of anti-inflammatory M2. Adiponectin reduces $\mathrm{T}$ cell responsiveness and $\mathrm{B}$ cell lymphopoiesis [48].

\section{Secreted frizzled-related protein 5 (SFRP5)}

SFRP5 is the anti-inflammatory adipocytokine, that binds to sequester pro-inflammatory wingless-type protein-member 5a (Wnt5a), prevents its binding to receptors, and this way, counteracts the inflammatory response induced by WNT5a. After the experimental induction of obesity in mice, a decrease SFRP5 concentration was observed, whereas, Wnt5a was increased. The Wnt5a/Sfrp5 protein expression ratio in AT in humans increases in obesity [49]. Sfrp5-knockout mice (Sfrp5-KO) have normal glucose tolerance on a regular diet, whereas on a HFD show impaired insulin sensitivity, severe glucose intolerance and increased fatty liver disease [50]. SFRP5 deficiency leads to exacerbation of metabolic dysfunction by increasing the accumulation of macrophages in white AT and enhancing the production of pro-inflammatory cytokines such as TNF- $\alpha$ and IL-6.

\section{Adipolin}

Adipolin (adipose-derived insulin-sensitizing factor) belongs to CRTP family proteins. It is mainly produced in 
adipocytes, and its expression in fat tissue and plasma is decreased in mice models of obesity [49]. Of note, systemic administration of adipolin to diet-induced obese mice is associated with decreased macrophage infiltration and reduced expression of pro-inflammatory adipocytokines in AT, which leads to improvement of glucose tolerance and insulin sensitivity [49].

\section{Special systemic conditions in obesity}

AT is considered as an endocrine organ, and is responsible for the production and secretion of many molecules including adipokines and inflammatory mediators that may exacerbate chronic inflammation in fat tissue, and affect the immunological activity of other cells and tissues. Several mechanisms are suggested to be responsible for chronic inflammation in obesity. There are: adipocytes' hypoxia, oxidative stress, endoplasmic reticulum stress, impaired PPAR receptors, inflammasomes' activation, increased adipocyte death and activation of TLR receptors.

\section{Adipocytes' hypoxia}

Hypoxia of obese AT, caused of rapid adipocyte expansion, and increased distance to oxygen, forces an increase in angiogenesis and tissue remodelling [51, 52]. In the low-oxygen environment adipose cells must adapt by altering gene expression patterns. Hypoxia-inducible factor-1 (HIF-1) is a transcription factor that increases during hypoxic episode, and results in increased transcription of genes involved in erythropoiesis and angiogenesis. Adipocytes' hypoxia leads to increased macrophage infiltration, and Th17 or Treg differentiation [52]. During hypoxic episode immune cells, especially macrophages, produce an increased amount of pro-inflammatory cytokines [51]. Hypoxia by up-regulation HIF1 gene transcription induces GLUT1 expression in macrophages, but it is adipose-specific rather than systemic. Hypoxia also inhibits the differentiation of preadipocytes into adipocytes and stimulates the polarization of macrophages into proinflammatory M1 macrophages.

\section{Oxidative stress}

Obesity dependent chronic inflammation is closely related to cellular oxidative stress. Increasing the level of free fatty acids and glucose in obesity, increases electron transport chain (ETC) activity in mitochondrias and active nicotinamide adenine dinucleotide phosphateoxidase (NADPH) causes an increased reactive oxygen species (ROS) production by adipocytes, which in turn leads to activation of the pro-inflammatory cascade. BMI is closely related to oxidative stress and ROS content in adipocytes [53]. Oxidative stress leads to an increased infiltration of macrophages into fat tissue. In addition, adipokines secreted by AT by activation of the NF- $\kappa \mathrm{B}$ contribute to the overproduction of ROS and increase oxidative stress, setting up a vicious circle [54].

\section{Endoplasmic reticulum stress}

Endoplasmic reticulum's (ER) function is close linked with metabolic homeostasis, and ER stress give the critical role of inflammation in obesity. In modified AT the synthesis of lipids and proteins increases and the balance between nutrients inside the cell is disturbed. This leads to impaired post-translational modifications of proteins and irregularities in their folding. The accumulation of misfolded proteins in the light of ER leads to the induction of ER stress and activation of the adaptive stress response pathway called the unfolded protein response (UPR), which aims to restore homeostasis in ER. An important role in the activation of the UPR pathway is played by three ER membrane-associated proteins, PERK (PKR-like eukaryotic initiation factor $2 \alpha$ kinase), IRE1 (inositol requiring enzyme 1), and ATF6 (activating transcription factor-6). In a situation where is no stress within the ER, these proteins are inactivated by the attachment of BiP chaperones, while under stress the BiP proteins are disconnected and three pathway are activated [55]. Experimental URP induction in cellular systems, causes increased expression of proinflammatory molecules such as IL-8, IL-6, MCP-1 and TNF $\alpha$ [56]. ER stress by activating of NF- $\kappa \mathrm{B}$ and JNK kinase, regulate transcription of inflammatory genes. Otherwise, ER stress can induce autophagy in cells, with several UPR pathways implicated in this interaction [57]. ER stress also enhances of proinflammatory adipokines secretion and impairs adipocytes' differentiation. One of the consequence of ER stress is the accumulation of ROS that promotes a state of oxidative stress.

\section{Peroxisome proliferator-activated receptors (PPAR)}

PPARs are ligand-dependent transcription factors belonging to a superfamily of nuclear hormone receptors, which control the expression of genes involved in development of inflammation and energy homeostasis. Their natural ligands are free fatty acids and eicosanoids. There are three types of PPAR: PPAR $\alpha$, PPAR $\beta / \delta$ and PPAR $\gamma$ receptors. PPAR $\alpha$ receptors are found in skeletal muscle, hepatocytes, cardiomyocytes and kidneys, where they affect the $\beta$-oxidation of fatty acids. These receptors have anti-inflammatory effects. In PPAR $\alpha$ knockout-mice on HFD is observed increased pro-inflammatory cytokine production in ATMs [58]. While PPARr occurs primarily in adipocytes and are involved in the regulation of adipocyte differentiation. PPARr agonists increase the sensitivity of adipocytes and muscle to insulin as well as glucose uptake and synthesis in these cells. PPARr ligands also cause a decrease of free fatty acids concentration by increasing their uptake and increasing the rate of fatty acid 
$\beta$-oxidation. PPARr are also found in many cells of the immune system: monocytes, macrophages, $\mathrm{T}$ and $\mathrm{B}$ lymphocytes, NK cells and dendritic cells. In particular, activation of PPARr by its agonists inhibits the expression of the $\mathrm{NF}-\kappa \mathrm{B}$ genes and proinflammatory molecules such as: NO, TNF $\alpha$, IL-6, IL-1 $\beta$, and IL-12. PPAR $\gamma$ are activated not only by ligand binding, but also by post-translational modifications (PTMs), including phosphorylation, SUMOylation, acetylation, and ubiquitination [59]. PPAR $\gamma$ together with PPAR $\beta / \delta$ lead to polarization of ATMs towards the M2 subtype and reduce inflammation in AT [10].

\section{Inflammasomes}

An important factor in the development of obesity-dependent inflammation is inflammasome. Martinon et al. in 2002 [60], for the first time have used the term inflammasomes for intracellular multi-protein complexes with an intrinsic ability to initiate an innate immune response upon the recognition of a pathogen-associated molecular patterns (PAMPS) or a damage-associated molecular patterns (DAMPS). These patterns are recognized by specialized structures, called pattern recognition receptors (PRR), in the cytoplasm, on the cell surface, or in endosomal compartment (e.g. Toll-like receptors - TLRs). Engagement of these PRRs triggers downstream signalling pathways leading to the production of proinflammatory cytokines such as IL1 $\beta$ or IL-18. Four classes of inflammasomes have been identified: the NLR family, pyrin domain-containing 1 and 3 (NLRP1 and NLRP3), CARD domain-containing 4 (NLRC4), and absent in melanoma 2 (AIM2) inflammasome. The NLRP3 inflammasome is the best characterized. Structurally it is composed of the NLR receptor (nucleotide-binding oligomerization domain (NOD)-like receptor), adaptor protein ASC (apoptosis-associated speck-like protein containing a carboxy-terminal CARD) and caspase-1. Many PAMPS and DAMPS, such as viruses, bacteria, toxins and extracellular ATP, activate NLRP3 inflammasomes. Activation of the NLRP3 leads to the transformation of inactive forms of proIL- $1 \beta$ and proIL-18 into active forms, via caspase 1 [61]. This activity could be implicated in inflammatory disease, autoimmune disease, infection, cancer, and metabolic disorders [61]. It has been shown that the expression of inflammasome components is increased in the AT of diet-induced or genetically obese mice [62]. In contrary, caloric restriction decreases the NLRP3, ASC, and IL-1 $\beta$ transcript levels in AT [62]. The NLRP3 inflammasome's components are identified in adipocytes and macrophages. Interestingly, mice on longterm HFD with whole-body ablation of NLRP3, ASC or caspase- 1 are protected from obesity and insulin resistance, which is associated with reduced IL- $1 \beta$ in adipose tissue and IL-18 in the blood and also with decreased level of T lymphocytes in AT [62]. In humans, blockade of IL-1 $\beta$ signalling improved glycemic control and reduced inflammatory markers [63].

\section{Toll-like receptors}

Obesity activates chronic inflammatory signalling pathways, at least in part, through the activation of Tolllike receptors (TLRs) in different tissues. TLRs belong to family of transmembrane proteins, that recognize PAMPs. Eleven different TLRs have been identified in humans [64]. The activation of TLRs, except TLR3, is mediated by an adapter protein (myeloid differentiation factor 88 ) and leads to the activation of various pro-inflammatory pathways. The activation process involves MAPK, JNK and IKK kinases and releases $\mathrm{NF}-\kappa \mathrm{B}$, resulting in expression of genes of pro-inflammatory cytokines, such as TNF- $\alpha$, IL-1 $\beta$, IL-6, IL- 8 and IL-12. TLR2 and TLR4 are the most important in the regulation of AT inflammation. Lack of these receptors significantly improves insulin sensitivity and reduces inflammation associated with obesity, both in AT and other tissues [65]. It has been discovered that an increase in the concentration of saturated fatty acids (SFAs) in obesity affects the activation of TLR4 in adipocytes and macrophages via c-Src and JNK kinases. In turn, unsaturated fatty acids (USFAs) do not show such an effect, and even decrease JNK kinase activation [66].

\section{Conclusions}

Morphological and functional changes appearing in AT during the development of obesity result in local and systemic inflammation. Released adipokines, cytokines, fatty acids and other factors affect the immunological activity of all tissues and cells, and chronic inflammation associated with obesity leads to many health complications, like cardiovascular and liver diseases A better understanding of the processes involved in developing and maintaining inflammation in obesity can provide new treatments for obesity-related disorders and its proper prophylaxis.

\section{The authors declare no conflict of interest.}

\section{References}

1. Heilbronn IK, Campbell IV (2008): Adipose tissue macrophages, low grade inflammation and insulin resistance in human obesity. Current Pharmaceutical Design 14: 1225-1230.

2. Winer S, Chan Y, Paltser G, et al. (2009): Normalization of obesity-associated insulin resistance through immunotheraphy. Nat Med 15: 921-929.

3. Kanneganti TD, Dixit VD (2012): Immunological complications of obesity. Nat Immunol 13: 707-712.

4. Harman-Boehm I, Bluher M, Redel H, et al. (2007): Macrophage infiltration into omental versus subcutaneous fat across different populations: effect of regional adiposity and the comorbidities of obesity. J Clin Endocrinol Metab 92: 2240-2247.

5. Weisberg SP, McCann D, Desai M, et al (2003): Obesity is associated with macrophage accumulation in adipose tissue. J Clin Investig 112: 1796-1808. 
6. Lumeng CN, Bodzin JL, Saltiel AR (2007): Obesity induces a phenotypic switch in adipose tissue macrophage polarization. J Clin Investig 117: 175-184.

7. Kwiecień I, Polubiec-Kownacka M, Dziedzic D, et al. (2019): CD163 and CCR7 as markers for macrophage polarisation in lung cancer microenvironment. Cent Eur J Immunol 44: 395-402.

8. Gordon S, Martinez FO (2010): Alternative activation of macrophages: mechanism and functions. Immunity 32: 593-604.

9. Sun S, Ji Y, Kersten S, et al. (2012): Mechanisms of inflammatory responses in obese adipose tissue. Annu Rev Nutr 32: 261-286.

10. Szanto A, Bakint BL, Nagy ZS, et al. (2010): STAT 6 transcription factor is a facilitator of the nuclear receptor PPAR $\gamma$-regulated gene expression in macrophages and dendritic cells. Immunity 33: 699-712.

11. Wong N, Fam BC, Cempako GR, et al. (2011): Deficiency in interferon- $\gamma$ results in reduced body weight and better glucose tolerance in mice. Endocrinology 152: 3690-3699.

12. Elgazar-Carmon V, Rudich A, Hadad N, et al. (2008): Neutrophils transiently infiltrate intra-abdominal fat early in the course of high-fat feeding. J Lipid Res 49: 1894-1903.

13. Ferrante AW Jr (2013): The immune cells in adipose tissue. Diabetes Obes Metab 15 (Suppl 3): 34-38.

14. Keskin Kurt R, Okyay AG, Hakverdi AU, et al. (2014): The effect of obesity on inflammatory markers in patients with PCOS: a BMI-matched case-control study. Arch Gynecol Obstet 290: 315-319.

15. Mărginean CO, Meliț LE, Ghiga DV, et al. (2019): Early Inflammatory status related to pediatric obesity. Front Pediatr 7: 241.

16. Spencer LA, Weller PF (2010): Eosinophils and Th2 immunity: contemporary insights. Immunol Cell Biol 88: 250256.

17. Wu D, Molofsky AB, Liang HE, et al. (2011): Eosinophils sustain adipose alternatively activated macrophages associated with glucose homeostasis. Science 332: 243-247.

18. Wensveen FM, Jelencic V, Valentic S, et al. (2015): NK cells link obesity-induced adipose stress to inflammation and insulin resistance. Nat Immunol 16: 376-385.

19. Dungan LS, McGuinness NC, Boon L, et al. (2014): Innate IFN-gamma promotes development of experimental autoimmune encephalomyelitis: a role for NK cells and M1 macrophages. Eur J Immunol 44: 2903-2917.

20. Schipper HS, Rakhshandehroo M, van de Graaf SF, et al. (2012): Natural killer T cells in adipose tissue prevent insulin resistance. J Clin Invest 122: 3343-3354.

21. Feuerer M, Herrero L, Cipoletta D, et al (2009): Lean, but not obese, fat is enriched for a unique population of regulatory $\mathrm{T}$ cells that affect metabolic parameters. Nat Med 15: 930-939.

22. Jagannathan-Bogdan M, McDonnell ME, Shin H, et al. (2011): Elevated proinflammatory cytokine production by a skewed $\mathrm{T}$ cell compartment requires monocytes and promotes inflammation in type 2 diabetes. J Immunol 186: 11621172 .

23. Zúńiga LA, Shen WJ, Joyce-Shaikh B, et al. (2010): IL-17 regulates adipogenesis, glucose homeostasis, and obesity. J Immunol 185: 6947-6959.

24. Kucharska AM, Pyrżak B, Demkow U (2015): Regulatory T cells in obesity. Adv Exp Med Biol 866: 35-40.

25. Nishimura S, Manabe I, Nagasaki M, et al. (2009): CD8+effector $\mathrm{T}$ cells contribute to macrophage recruitment and adipose tissue inflammation in obesity. Nat Med 15: 914-920.
26. Cava L (2017): Leptin in inflammation and autoimmunity. Cytokine 98: 51-58.

27. DeFuria J, Belkina AC, Jagannathan-Bogdan M, et al. (2013): B cells promote inflammation in obesity and type 2 diabetes through regulation of $\mathrm{T}$ cell function and an inflammatory cytokine profile. PNAS 110: 5133-5138.

28. Molofsky AB, Nussbaum JC, Liang HE, et al. (2013): Innate lymphoid type 2 cells sustain visceral adipose tissue eosinophils and alternatively activated macrophages. J Exp Med 210: 535-549.

29. Brestoff JR, Kim BS, Saenz SA, et al. (2015): Groupe 2 innate lymphoid cells promote beiging of white adipose tissue and limit obesity. Nature 519: 242-246.

30. Good M, Newell FM, Haupt LM, et al. (2006): TNF and TNF receptor expression and insulin sensitivity in human omental and subcutaneous adipose tissue - influence of BMI and adipose distribution. Diab Vasc Dis Res 3: 26-33.

31. Hotamisligil GS, Shargil NS, Spiegelman BM (1993): Adipose expression of tumor necrosis factor-alpha: direct role in obesity-linked insulin resistance. Science 259: 87-91.

32. Kern PA, Saghizadehh M, Ong JM, et al. (1995): The expression of tumor necrosis factor in human adipose tissue. Regulation by obesity, weight loss, and relationship to lipoprotein lipase. J Clin Investig 95: 2111-2119.

33. Fontana L, Eagon JC, Trujillo ME, et al. (2007): Visceral fat adipokine secretion is associated with systemic inflammation in obese humans. Diabetes 56: 1010-1013.

34. Sopasakis VR, Sandqvist M, Gustafson B, et al. (2004): High local concentrations and effects on differentiation implicate interleukin-6 as a paracrine regulator. Obesity Res 12: 454460.

35. Warnberg J, Moreno LA, Mesana MI, et al. (2004): Inflammatory mediators in overweight and obese Spanish adolescents. The AVENA Study Int J Obes 28: S59-63.

36. Visser M, Bouter LM, McQuillan GM, et al. (2001): Lowgrade systemic inflammation in overweight children. Pediatrics 107: E13.

37. Rumińska M, Witkowska-Sędek E, Artemniak-Wojtowicz D, et al. (2019): Changes in leukocyte profile and C-reactive protein concentration in overweight and obese adolescents after reduction of body weight. Cent Eur J Immunol 44: 307-315.

38. Bennett NR, Ferguson TS, Bennett FI, et al. (2014): High-sensitivity C-reactive protein is related to central obesity and the number of metabolic syndrome components in Jamaican young adults. Front Cardiovasc Med 1: 12.

39. Gorska E, Popko K, Stelmaszczyk-Emmel A, et al. (2010): Leptin receptors. Eur J Med Res 15 (Suppl 2): 50-54.

40. Matarese G, Moschos S, Mantzoros CS (2005): Leptin in immunology. J Immunol 174: 3137-3142.

41. Makki K, Froguel P, Wolowczuk I (2013): Adipose Tissue in Obesity-Related Inflammation and Insulin Resistance: Cells, Cytokines, and Chemokines. ISRN Inflammation 2013: 139239.

42. Bokarewa M, Nagaew I, Dahlberg I, et al. (2005): Resistin, an adipokine with potent proinflammatory properties. J Immunol 174: 5789-5795.

43. Tabata M, Kadomatsu T, Fukuhara S, et al. (2009): Angiopoietin-like protein 2 promotes chronic adipose tissue inflammation and obesity-related systemic insulin resistance. Cell Metab 10:178-188.

44. Ryo M, Nakamura T, Kihara S, et al. (2004): Adiponectin as a biomarker of the metabolic syndrome. Circ J 68: 975-981. 
45. Ouchi N, Kihara S, Funahashi T, et al. (2003): Obesity, adiponectin and vascular inflammatory disease. Curr Opin Lipidol 14: 561-566.

46. Hosogai N, Fukuhara A, Oshima K, et al. (2007): Adipose tissue hypoxia in obesity and its impact on adipocytokine dysregulation. Diabetes 56: 901-911.

47. Maeda N, Shimomura I, Kishida K, et al. (2002): Dietinduced insulin resistance in mice lacking adiponectin/ACRP. Nature Medicine 8: 731-737.

48. Yokota T, Oritani K, Takahashi I, et al. (2000): Adiponectin a new member of the family of soluble defense collagens, negatively regulates the growth of myelomonocytic progenitors and the function of macrophages. Blood 96: 1723-1732.

49. Ouchi N, Ohashi K, Shibata R, Murohara T (2012): Adipocytokines and obesity-linked disorders. Nagoya J Med Sci 74: 19-30.

50. Ouchi N, Higuchi A, Ohashi K, et al. (2010): Sfrp5 is an antiinflammatory adipokine that modulates metabolic dysfunction in obesity. Science 329: 454-457.

51. O’Rourke RW, White AE, Metcalf MD, et al. (2011): Hypoxia-induced inflammatory cytokine secretion in human adipose tissue stromovascular cells. Diabetologia 54: 1480-1490.

52. Rausch ME, Weisberg S, Vardhana P, et al. (2008): Obesity in C57BL/6 $\mathrm{J}$ mice is characterized by adipose tissue hypoxia and cytotoxic T-cell infiltration. Int J Obes (Lond) 32: 451463.

53. Furukawa S, Fujita T, Shimabukuro M, et al. (2004): Increased oxidative stress in obesity and its impact on metabolic syndrome. J Clin Invest 114: 1752-1761.

54. Nunn AV, Bell JD, Guy GW (2009): Lifestyle-induced metabolic inflexibility and accelerated ageing syndrome: insulin resistance, friend or foe? (Lond) Nutr Metab 6: 16

55. Bertolotti A, Zhang Y, Hendershot LM, et al. (2000): Dynamic interaction of BiP and ER stress transducers in the unfolded-protein response. Nat Cell Biol 2: 326-332.

56. Li Y, Schwabe RF, DeVries-Seimon T, et al. (2005): Free cholesterol-loaded macrophages are an abundant source of tumor necrosis factor-alpha and interleukin-6: model of NF-kappaB- and map kinase-dependent inflammation in advanced atherosclerosis. J Biol Chem 280: 21763-21772.

57. Kouroku Y, Fujita E, Tanida I, et al. (2007): ER stress (PERK/eIF2alpha phosphorylation) mediates the polyglutamine-induced LC3 conversion, an essential step for autophagy formation. Cell Death Differ 14: 230-239.

58. Stienstra R, Mandard S, Patsouris D, et al. (2007): Peroxisome proliferator-acivated receptor $\alpha$ protects against obesity-induced hepatic inflammation. Endocrinology 148: 2753-2763.

59. van Beekum O, Fleskens V, Kalkhoven E (2009): Posttranslational modifications of PPAR-gamma: fine-tuning the metabolic master regulator. Obesity 17: 213-219.

60. Martinon F, Burns K, Tschopp J (2002): The inflammasome: a molecular platform triggering activation of inflammatory caspases and processing of proIL-beta. Mol Cell 10: 417-426.

61. Davis BK, Wen H, Ting JP (2011): The inflammasome NLRs in immunity, inflammation, and associated disease. Annu Rev Immunol 29: 707-735.

62. Vandanmagsar B, Youm YH, Ravussin A, et al. (2011): The NLRP3 inflammasome instigates obesity-induced inflammation and insulin resistance. Nat Med 17: 179-188.

63. Larsen CM, Faulenbach M, Vaag A, et al. (2007): Interleukin-1 receptor antagonist in type 2 diabetes mellitus. $\mathrm{N}$ Engl J Med 356: 1517-1526.
64. [64].Trudler D, Farfara D, Frenkel D (2010): Toll-like receptors expression and signalling in glia cells in neuroamyloidogenic diseases: Towards future therapeutic application. Mediators Inflamm 2010:497987.

65. Shi H, Kokoeva MV, Inouye K, et al. (2006): TLR4 links innate immunity and fatty acid-induced insulin resistance. J Clin Invest 116: 3015-3025.

66. Könner AC, Brüning JC (2011): Toll-like receptors: linking inflammation to metabolism. Trends Endocrinol Metab 22: 16-23. 